\title{
SURGICAL MANAGEMENT OF INTESTINAL ATRESIA AND OBSTRUCTION IN NEONATES - OUR EXPERIENCE
}

\author{
Moka Jagan Mohan'1, Thatipamula Anil Balraj², Tanuja K. D. B. S \\ ${ }_{1}^{1}$ Associate Professor, Department of Paediatric Surgery, Andhra Medical College, Visakhapatnam, Andhra Pradesh, India. \\ ${ }^{2}$ Associate Professor, Department of Paediatric Surgery, Osmania Medical College, Hyderabad, Telangana, India. \\ ${ }^{3}$ Postgraduate Student, Department of General Surgery, Kakatiya Medical College, Warangal, Telangana, India.
}

\section{ABSTRACT}

\section{BACKGROUND}

The neonatal period is defined as the first four weeks after birth. Intestinal atresia is the commonest cause of intestinal obstruction in the new born children. It requires early recognition and prompt treatment to obtain better outcome.

The aim of the present study was to focus on aetiology, diagnosis and surgical management of intestinal atresia and obstruction in neonates.

\section{MATERIALS AND METHODS}

This study was done retrospectively and included all operated cases of intestinal atresia and obstruction during neonatal period (first 4 weeks after birth) over a period of 4 years from January 2012 to December 2015 from the Department of Paediatric Surgery in MGM Hospital/Kakatiya Medical College, Warangal, Telangana state. We have studied their clinical presentation, diagnosis, surgical management and their outcome.

\section{RESULTS}

A total of 42 cases were included in this study. Female-male ratio was 1.3:1. Gestational age was from 32 to 40 weeks (Mean=34.2 weeks). Birth weight was $1.5 \mathrm{~kg}$ to $3.0 \mathrm{~kg}$ (Mean=2.0 kg). We had seen 4 cases of Duodenal atresias, 19 cases of Jejunoileal atresias, 11 cases of malrotation of gut, 3 cases of meconium ileus, 3 cases of NNEC (Neonatal Necrotising Enterocolitis) with intestinal perforation, 1 case of volvulus due to mesenteric cyst and 1 case of patent vitello intestinal duct. We had taken bilious aspirate as the diagnostic criteria to diagnose the intestinal obstruction. Mortality rate was $26.19 \%$ mostly due to prematurity and septicaemia in pre- and post-operative period.

\section{CONCLUSION}

Although the morbidity and mortality was very high in neonatal intestinal obstruction, this has improved in the current era by improved care taken in sick newborn care unit (SNCU), early detection of cases by antenatal scan and proper adequate resuscitation before and after surgery. Biliary aspirate should be always suspected with intestinal obstruction and should be investigated thoroughly to rule out the same.

\section{KEYWORDS}

Intestinal Atresia, Intestinal Obstruction, Malrotation of Gut, Meconium Ileus.

HOW TO CITE THIS ARTICLE: Mohan MJ, Balraj TA, Tanuja KDBS. Surgical management of intestinal atresia and obstruction in neonates - Our experience. J. Evolution Med. Dent. Sci. 2017;6(2):74-78, DOI: 10.14260/Jemds/2017/19.

\section{BACKGROUND}

Performing surgery in a newborn child is a challenge to a paediatric surgeon in developing countries like India. These patients require management in SNCU (Sick Newborn Care Unit) or NICU (Neonatal Intensive Care Unit) with a team of well-equipped paediatric nursing care, well-experienced paediatric surgeon and paediatric anaesthesiologist to get better survival rates. ${ }^{1}$ Only few studies are available on neonatal intestinal obstruction in Indian literature.

In neonates, intestinal obstruction is the most common surgical emergency which requires immediate attention. ${ }^{2}$ The

Financial or Other, Competing Interest: None.

Submission 16-12-2016, Peer Review 29-12-2016,

Acceptance 31-12-2016, Published 05-01-2017.

Corresponding Author:

Dr. Thatipamula Anil Balraj,

3-12-20/B/302, SK Viviaana Apartments,

Sri Laxmi Nagar Colony,

Mansoorabad Road, LB Nagar,

Hyderabad-500068.

E-mail: dr.sunanda.anil@gmail.com

DOI: $10.14260 /$ jemds $/ 2017 / 19$ incidence is estimated 1 in 2,000 live births. The cardinal signs of intestinal obstruction are history of maternal polyhydramnios, feeding intolerance, bilious vomiting, delayed passage of meconium in first 24 hours after birth, abdominal distension and visible loops of bowel. ${ }^{3}$ The common causes are duodenal atresia, intestinal atresia, meconium ileus, malrotation of gut with or without volvulus. An accurate history and physical examination along with simple radiologic studies like plain X-ray abdomen usually leads to the correct diagnosis. This X-ray should be taken in erect and supine views which often will give a clue to the site of obstruction. Mortality and morbidity is reduced by early diagnosis and prompt treatment.4,5 Delay in diagnosis of neonatal bowel obstruction leads to bilious aspiration, sepsis, infarction of the midgut due to volvulus and thus increased mortality. Other factors contributing to the mortality in developing countries include prematurity, late presentation, associated congenital anomalies and lack of intensive care facilities. ${ }^{2,6,7}$ 


\section{Aim of the Study}

The aim of the present study was to find out the different types of presentation of neonatal intestinal obstruction, surgical management and its outcome in the Paediatric Surgery Department at MGM Hospital, Kakatiya Medical College which is a district level teaching hospital in Warangal.

\section{MATERIALS AND METHODS}

This retrospective study was conducted in Department of Paediatric Surgery, MGM Hospitals, Warangal. Over the last 4 years period from January 2012 to December 2015, all the neonates with intestinal obstruction who were referred from SNCU for surgical opinion and management were studied. Out of these newborns, 42 patients who underwent surgery for intestinal obstruction were included in this study.

The patients who were very sick, who are on ventilator and unfit for anaesthesia and surgery were excluded from our study. Neonates with oesophageal atresia, Congenital Hypertrophic Pyloric Stenosis (CHPS), Hirschsprung's disease and anorectal malformations were also excluded from this study, because presence of bilious aspirate was taken as the criteria for intestinal atresia and obstruction.

Data were collected from the hospital records and analysed for age of the baby at the time of presentation, sex, clinical presentation, provisional diagnosis, investigations, the type of surgical procedure performed and its outcome.

All patients were managed by IV fluids, antibiotics and nasogastric or orogastric decompression preoperatively. Dehydration and electrolyte imbalance were corrected preoperatively. All of them underwent routine blood investigations and plain X-ray abdomen in erect and supine position (Figure1).

Special studies like oral contrast meal or enema were performed in 14 patients to diagnose malrotation and meconium ileus. Oral contrast meal showed dilated stomach and duodenum with DJ flexure and jejunal loops on to the right side of spine. Ultrasound was performed in one patient which showed mesenteric cyst leading to volvulus of the small bowel.

\section{RESULTS}

We have analysed 42 cases of intestinal obstruction in neonates in the Department of Paediatric Surgery, MGM Hospitals, Warangal. Female:male ratio was 1.3:1. Most of the neonates were diagnosed within 2 weeks after birth (Table No-1). Most common cause of intestinal obstruction was jejunoileal atresia (19 patients), malrotation of the gut (11cases), duodenal atresia(4 cases), meconium ileus (3 cases), neonatal necrotising enterocolitis with perforation (3 cases), volvulus due to mesenteric cyst or duplication cyst(1 case) and one case of Patent Vitello Intestinal Duct (Table No-2).

Bilious vomiting was the cardinal feature of intestinal atresia and obstruction. Visible loops of intestine were observed on per abdominal examination with passage of mucus pellets in the stools in intestinal atresia (19 cases). Plain X-ray abdomen showed air-fluid levels (Fig: 1). In malrotation of gut (11 cases) contrast meal was helpful in diagnosis as they showed dilatation of the stomach and duodenal bulb with DJ flexure and jejunal loops on to the right side of spine (Fig: 2). Plain X-ray abdomen showed double bubble sign in 4 patients and were diagnosed as duodenal atresia (Fig: 3). Three cases of NNEC presented with distension of abdomen and pneumoperitoneum. One out of 3 cases of meconium ileus presented as pneumoperitoneum due to perforation in dilated ileum. One case of intestinal obstruction had palpable lump diagnosed to be a mesenteric cyst by ultrasound was confirmed during surgery. One patient presented with prolapsed ileum from the umbilicus with signs of obstruction (Fig. 4). All the cases were operated after obtaining the high risk consent from the parents of patients. Haemodynamic and fluid-electrolyte balance was corrected pre-operatively and exploratory laparotomy was performed. Appropriate procedure was decided on the operating table during laparotomy.

We performed resection of dilated segment and end to back anastomosis in all the cases of jejunoileal atresia. Bishop-Koop procedure was performed in 5 patients who developed leak from the anastomotic site, out of which 4 patients died and one survived. The ileostomy was closed after one year when the baby was healthy and fit for surgery. In two patients, Santulli procedure was done after leak from anastomosis, out of which one patient survived and other expired due to septicaemia.

One patient was presented with intestinal atresia where all types of anomalies (type I to IV) were observed. In this patient, about $25-30 \mathrm{~cm}$ of the intestine was resected and end-to-end anastomosis was performed. Postoperative period was uneventful and patient was discharged on $10^{\text {th }}$ post-operative day.

All 5 cases of duodenal atresias were operated and duodenoduodenostomy was performed. In one of the patient, we observed the association of duodenal atresia with oesophageal atresia, developed leak from oesophagus postoperatively and died due to septicaemia. In 9 cases of malrotation of gut, Ladd's procedure was performed. Resection of the midgut and anastomosis of proximal jejunum with distal ileum was performed in two cases where gangrenous bowel was observed during surgery. One patient expired on the second post-operative day. Short bowel syndrome was developed in another patient 2 weeks after the surgery and later expired due to aspiration.

Bishop-Koop ileostomy was performed in all three cases of meconium ileus. One patient died after 2 days postoperatively due to prematurity and hypothermia.

All three cases of neonatal necrotising enterocolitis with perforation were operated. Resection of gangrenous part and exteriorisation of the bowel was performed in two cases, but both of them expired due to septicaemia. Resection and anastomosis was performed in one case which survived.

\begin{tabular}{|c|c|c|c|}
\hline Age & Male & Female & Total \\
\hline 0-7 days & 9 & 8 & 17 \\
\hline 8-14 days & 5 & 10 & 15 \\
\hline 15-21days & 3 & 1 & 4 \\
\hline 21-28days & 2 & 4 & 6 \\
\hline Total & $\mathbf{1 9}$ & $\mathbf{2 3}$ & $\mathbf{4 2}$ \\
\hline \multicolumn{4}{|l}{ Table 1. Demographic Distribution } \\
\hline
\end{tabular}




\begin{tabular}{|c|c|c|c|c|}
\hline $\begin{array}{c}\text { Sl. } \\
\text { No. }\end{array}$ & Aetiology & $\begin{array}{c}\text { No. of } \\
\text { Patients }\end{array}$ & $\begin{array}{c}\text { No. of } \\
\text { Deaths }\end{array}$ & $\begin{array}{c}\text { Percent- } \\
\text { age }\end{array}$ \\
\hline 1 & Duodenal Atresia & 04 & 01 & $25 \%$ \\
\hline 2 & Jejunoileal atresia & 19 & 06 & $31.57 \%$ \\
\hline 3 & Malrotation of Gut & 11 & 02 & $18.18 \%$ \\
\hline 4 & Meconium ileus & 03 & 01 & $33.33 \%$ \\
\hline 5 & $\begin{array}{c}\text { NNEC (Neonatal } \\
\text { Necrotising } \\
\text { Enterocolitis) }\end{array}$ & 03 & 02 & $66.66 \%$ \\
\hline 6 & $\begin{array}{c}\text { Volvulus due to } \\
\text { mesenteric cyst }\end{array}$ & 01 & ---- & ----- \\
\hline 7 & $\begin{array}{c}\text { Patent Vitello Intestinal } \\
\text { Duct }\end{array}$ & 01 & ------ & ----- \\
\hline \multicolumn{4}{|c|}{ Table 2. Distribution of Patients by Aetiology } \\
\hline
\end{tabular}

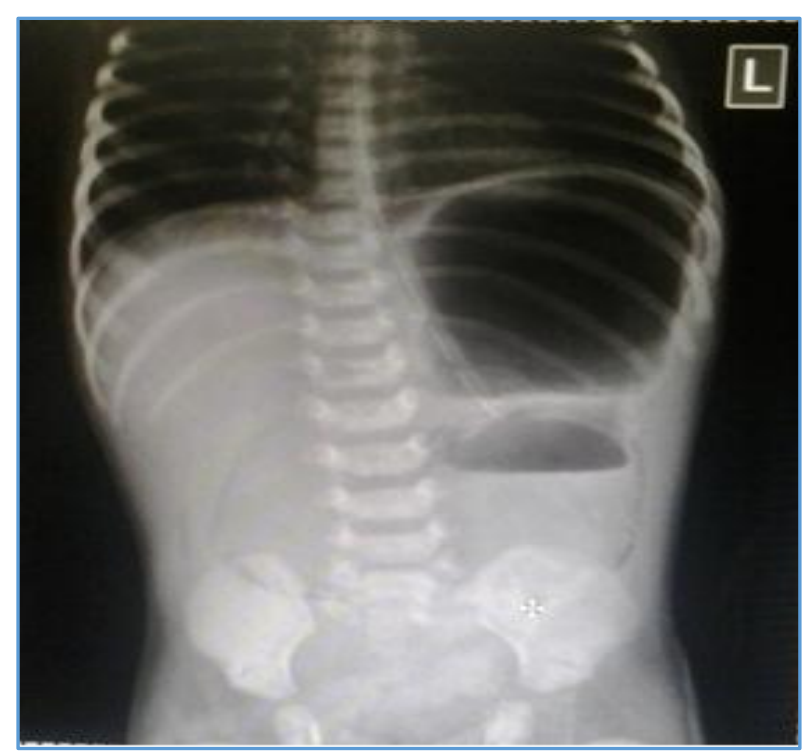

Figure 1. X-ray Abdomen Erect showing Dilated Stomach and Jejunum as fluid levels one below the other

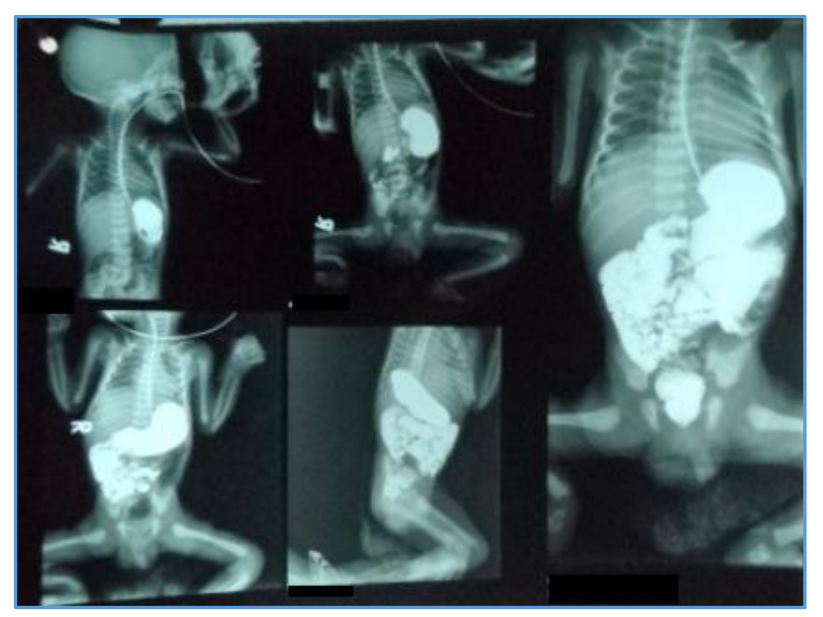

Figure 2. Contrast Meal X-rays showing Dilated Stomach and DJ flexure and Jejunal loops on right side suggestive of Malrotation of Gut

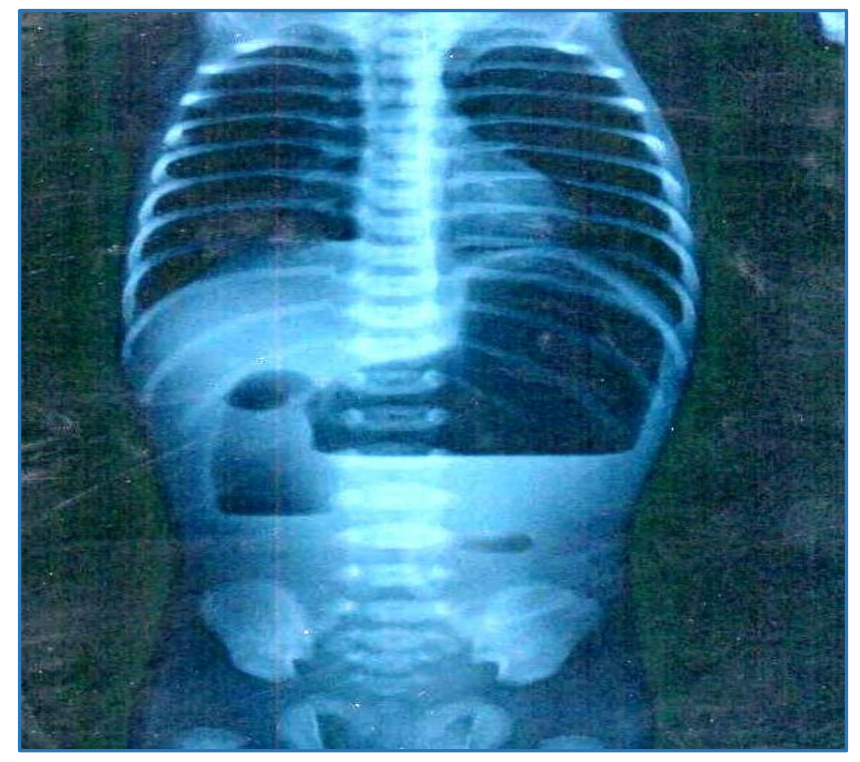

Figure 3. Plain X-ray Abdomen showing Double Bubble sign in Duodenal Atresia

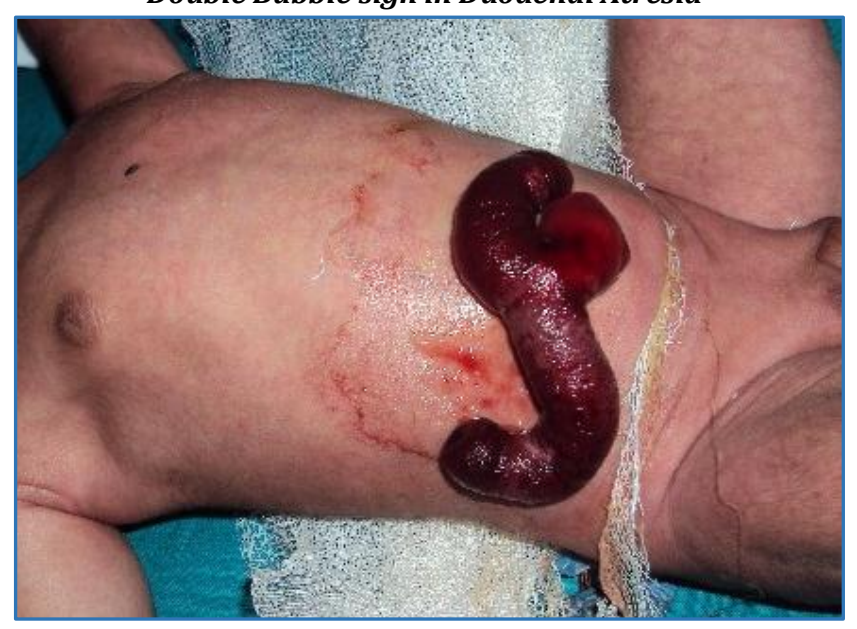

Figure 4. Patent VID seen as Prolapsed Ileum from the Umbilicus with Obstruction

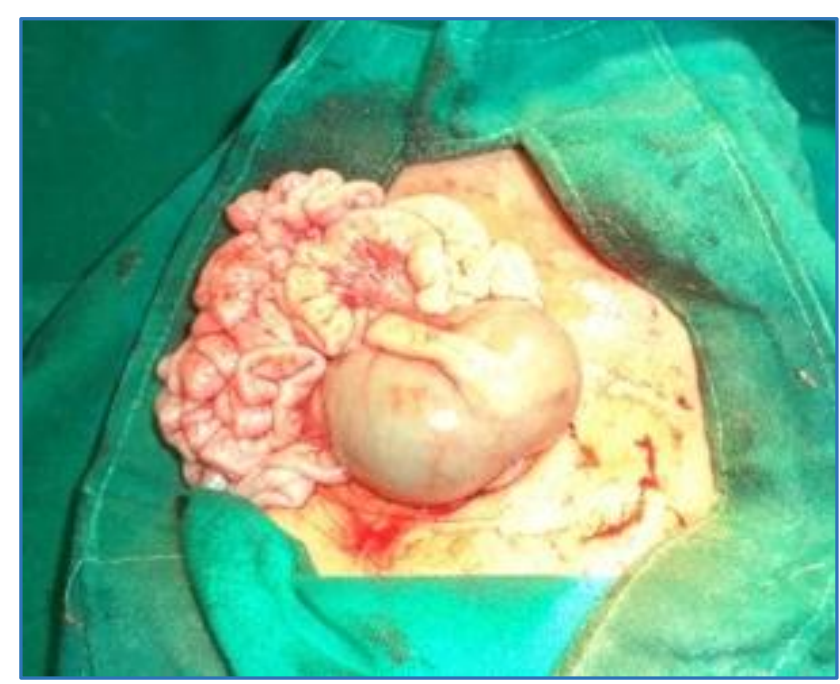

Figure 5. Proximal Jejunal Atresia Type 1-Diaphragm as seen at Operation 


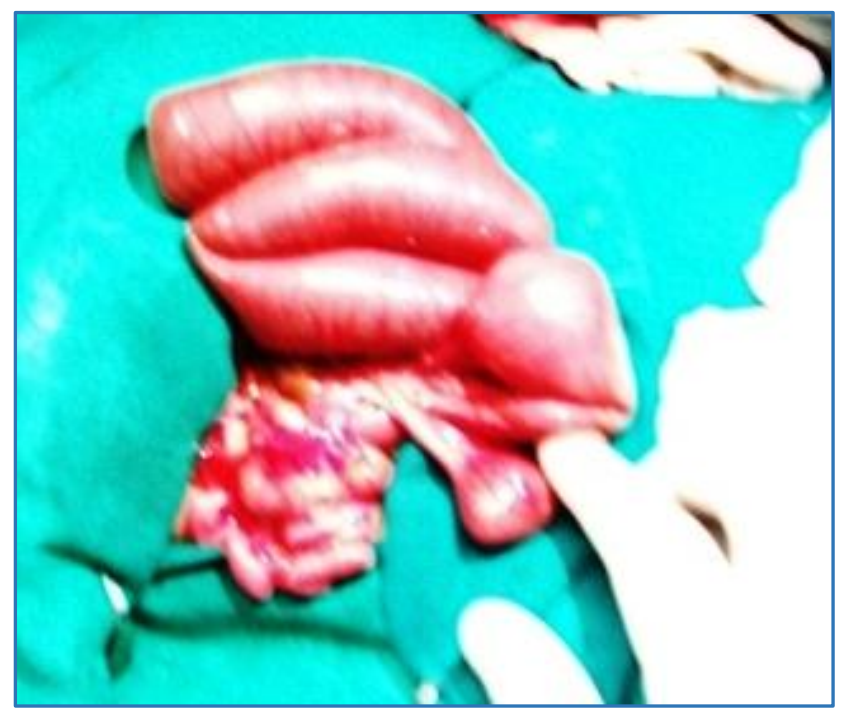

Figure 6. Type 3a Atresia with Defect in Mesentery

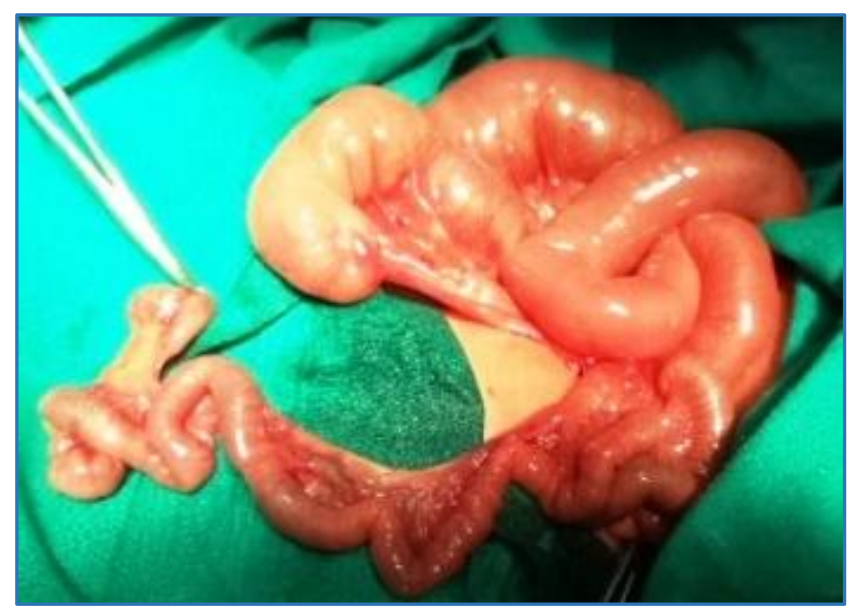

Figure 7. Type 3 b Atresia Apple peel Atresia

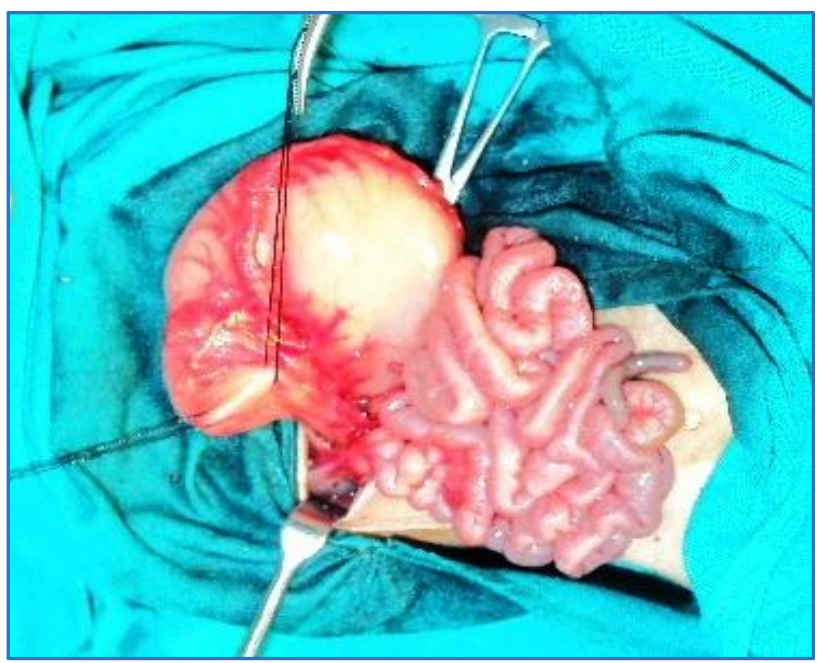

Figure 8. Duodenal Atresia showing Dilated Stomach Proximally and Atretic Duodenum Distally

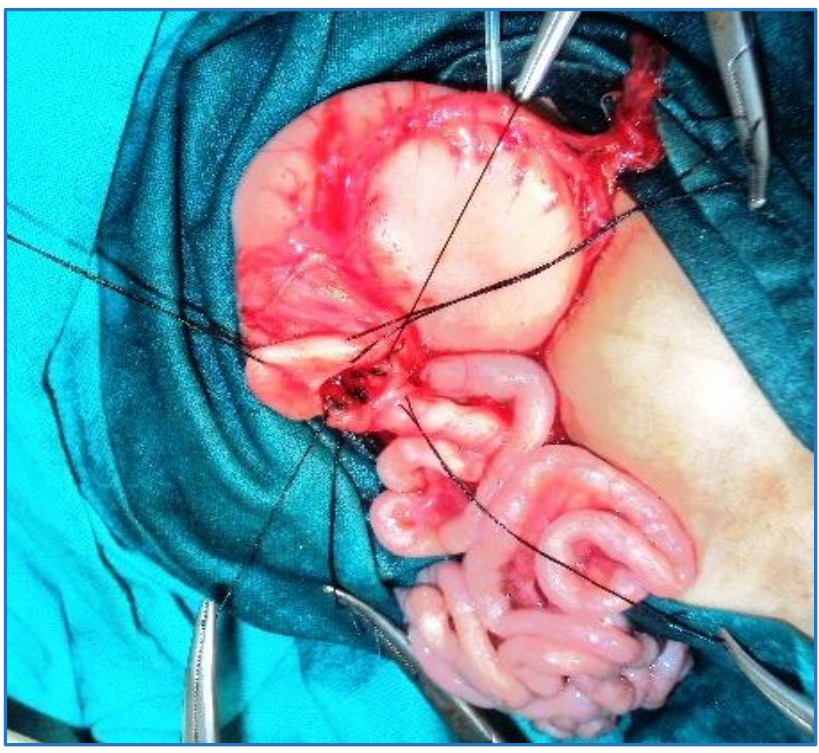

Figure 9. Intra-op Picture showing the Diamond shaped Anastomosis in Duodenal Atresia

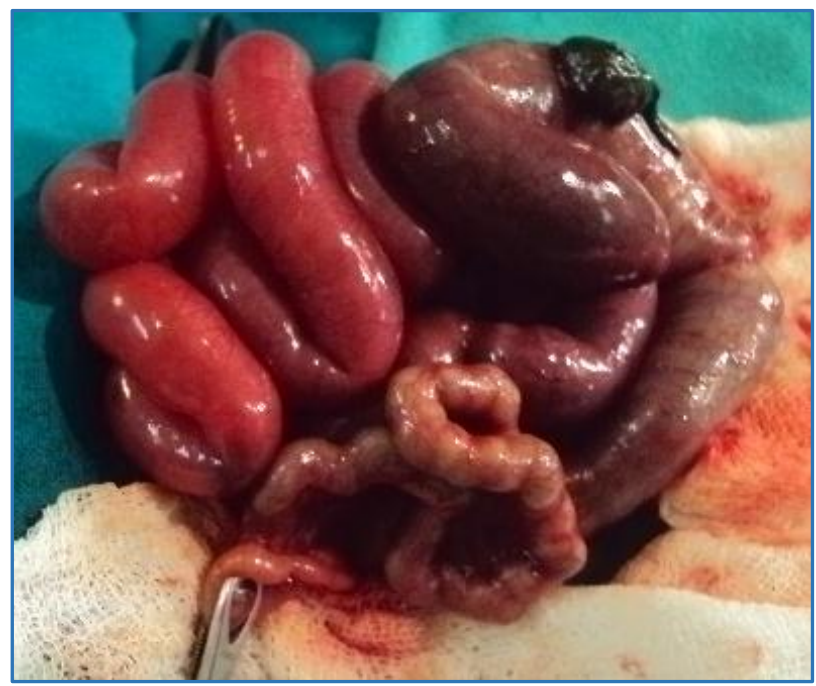

Figure 10. Intra-op Picture showing Meconium Ileus with Perforation in Dilated Ileum and Pellets in Distal Ileum

\section{DISCUSSION}

The most common cause of intestinal obstruction in neonates is intestinal atresia. Out of which jejunal and duodenal atresia are much more common.

Atresias are classified into 5 types.

- Type I - Membrane or diaphragm causing obstruction in the lumen (Fig: 5),

- Type II - A cord type of anomaly at the site of atresia with intact mesentery,

- Type IIIA - Which involves separation of both ends by a mesenteric defect (Fig: 6),

- Type IIIB- Apple-peel deformity where the distal loop is coiled and supplied by the ileocolic artery (Fig: 7),

- $\quad$ Type IV - Multiple intestinal atresia. ${ }^{8}$

Duodenal atresias are due to failure of recanalisation that normally occurs during 9-11 weeks of gestational age. It is commonly associated with various anomalies such as Down syndrome most often seen, CHD, as part of VACTERL 
association (As we have seen in one case of oesophageal atresia) additional intestinal atresia. ${ }^{9}$ It is treated by duodenoduodenostomy with linear or diamond shaped. We did diamond shaped anastomosis in all our cases (Fig: 8 \& 9).

Malrotation of the gut is the next common cause of intestinal obstruction. It is caused by a failure of rotation of the normal bowel during entry of bowel into the abdomen from yolk sac. Bilious vomiting is the diagnostic criteria for diagnosing these cases. More than $50 \%$ of them present during neonatal period with history of bilious vomiting. However, few of the patients manifest in teenagers and adults, often with dangerous outcome and difficulty in diagnosis. Many children with malrotation become symptomatic before the age of 1 year, some develop symptoms later and few may never have symptoms in their whole life. Symptoms happen if the intestine gets blocked (Such as by Ladd's bands or volvulus) or if blood flow to the intestines is cut off. 10

Upper GI contrast studies are both sensitive and accurate in diagnosing malrotation seen as DJ flexure on to the right side of the spine and jejunal loops are seen in RIF with whorl sign. There is dilatation of stomach and duodenum. Ladd's procedure is the treatment of choice which includes derotation of the volvulus. Lysis of Ladd's bands with Kocherisation of the duodenum along the ascending colon with or without appendicectomy and placing the caecum into the left iliac fossa. We performed appendicectomy in all our cases as it may pose diagnostic difficulties in future attacks of appendicitis. ${ }^{11}$

Patients with meconium ileus presented with bilious vomiting and gross abdominal distension due to obstruction of the terminal ileum by thick meconium. Plain X-ray abdomen shows absence of air/fluid levels and ground glass appearance. The treatment of choice for meconium ileus was non-operative hyperosmolar Gastrografin or Omnipaque enema which works as diagnostic and therapeutic procedure. In complicated cases with perforation, it requires surgical resection and primary anastomosis or sometimes by an ileostomy such as Bishop-Koop or Santulli procedure. ${ }^{12} \mathrm{We}$ performed Bishop-Koop procedure in all our meconium ileus cases (Fig: 10).

Neonatal necrotising enterocolitis is one the most common gastrointestinal emergencies seen in SNCU. The aetiopathology of NNEC still remains unclear despite of research. The mainstay of surgical management is resection of the gangrenous portion of the intestine and enterostomy. Occasionally, end-to-end anastomosis can be performed in few selected cases. In some cases, only peritoneal drainage was performed first and second look surgery or proximal diversion is performed next. ${ }^{13}$

One patient had duplication cyst of ileum as mesenteric cyst with localised volvulus of ileum causing obstruction. We did resection of the bowel along with the cyst and end-to-end anastomosis. Patient recovered well after surgery.
Patent Vitello Intestinal Duct presented as prolapse of ileum from the umbilicus ( 1 patient). Resection and end-toend anastomosis was performed and patient recovered well.

\section{CONCLUSION}

Our study showed that intestinal atresia followed by malrotation of the gut are the most common causes of neonatal intestinal obstruction. Bilious vomiting was one of the cardinal feature in both the conditions. Radiology in the form of plain X-ray abdomen and contrast studies helped in the early diagnosis and treatment.

Antenatal diagnosis with early referral and proper care in SNCU by well-trained staff and well-trained paediatric surgical team in neonatal surgery are all necessary to reduce morbidity and mortality in ensuring better outcomes in treating neonatal intestinal obstruction.

\section{REFERENCES}

[1] Spitz L. The management of neonatal surgical emergencies. In: Taylor I. (edtr.) Progress in surgery. Vol 2. $15^{\text {th }}$ edn. Churchill Livingstone, Edinburgh 1987:18-33.

[2] Seth A, Chanchlani R, Rakhonde AK. Neonatal gastrointestinal emergencies in a tertiary care centre in Bhopal, India: a prospective study. IJSS 2015;1(2).

[3] Juang D, Snyder CL. Neonatal bowel obstruction. Surg Clin North America 2012;92(3):685-711.

[4] De la Hunt MN. The acute abdomen in the newborn. Semin Fetal Neonatal Med 2006;11(3):191-7.

[5] De Silva NT, Young JA, Wales PW. Understanding neonatal bowel obstruction: building knowledge to advance practice. Neonatal Netw 2006;25(5):303-18.

[6] Ameh EA, Chirdan LB. Neonatal intestinal obstruction in Zaria, Nigeria. East Afr Med J 2000;77(9):510-3.

[7] Uba AF, Edino ST, Yakubu AA, et al. Childhood intestinal obstruction in North western Nigeria. West Afr J Med 2004;23(4):314-8.

[8] Hajivassiliou CA. Intestinal obstruction in neonatal/pediatric surgery. Seminars in Pediatric Surgery 2003;12(4):241-53.

[9] Rowe MI. Essentials of paediatric surgery. Mosby year book. Inc 1995:pp 899.

[10] Dietz DW, Walsh RM, Grundfest-Broniatowski S, et al. Intestinal malrotation: a rare but important cause of bowel obstruction in adults. Dis Colon Rectum 2002;45(10):1381-6.

[11] Torres AM, Ziegler MM. Malrotation of the intestine. World J Surg 1993;17(3):326-31.

[12] Nasir GA, Rahma S, Kadim AH. Neonatal intestinal obstruction. Eastern Mediterranean Health Journal 2000;6(1):187-93.

[13] Neu J, Walker WA. Necrotizing enterocolitis. N Engl J Med 2011;364(3):255-64. 